\title{
Das Gesetz zur Verbesserung der Versorgungsstrukturen in der gesetzlichen Krankenversicherung - GKV-Versorgungsstrukturgesetz
}

CHRISTIAN PETERS, BIRGIT SCHLIEMANN UND STEPHAN FELDMANN

Dr. Christian Peters, MBA ist Leiter der Abteilung für Ambulante Versorgung des AOK-Bundesverbandes

Birgit Schliemann, MPH ist Mitarbeiterin der Abteilung für Ambulante Versorgung des AOK-Bundesverbandes

Stephan Feldmann ist Mitarbeiter der Abteilung für Ambulante Versorgung des AOK-Bundesverbandes
"Mit dem Versorgungsstrukturgesetz ebnen wir den Weg zu einer langfristigen qualitativ hochwertigen medizinischen Versorgung", so der Bundesminister für Gesundheit optimistisch. Um demographischer Entwicklung und heterogener Verteilung von ambulanten Versorgungsangeboten in der Fläche zu begegnen, sieht das Gesetz die Rücknahme zentraler Vorgaben bei der vertragsärztlichen Vergütung, eine Stärkung der Aufsichts- und Beteiligungsrechte der Länder in der Bedarfsplanung sowie die Schaffung eines neuen "spezialfachärztlichen" Versorgungsbereichs für ausgewählte Erkrankungen vor. Die Erwartungen an eine verbesserte Integration der Sektoren sowie eine höhere Attraktivität der Niederlassung in strukturschwachen Gebieten waren im Vorfeld des Gesetzgebungsverfahrens hoch: Kann das Versorgungsstrukturgesetz sie tatsächlich erfüllen?

\section{Entstehungsgeschichte}

Der Bundesrat hat am 16. Dezember das zu Beginn des Monats von der schwarzgelben Regierungskoalition im Bundestag verabschiedete »Gesetz zur Verbesserung der Versorgungsstrukturen in der gesetzlichen Krankenversicherung « (»GKV-Versorgungsstrukturgesetz « GKV-VStG) gebilligt und damit einen vorläufigen Schlusspunkt hinter die Entstehung eines komplexen Gesetzeswerks gesetzt, das mehr als ein Jahr lang Anlass intensiver Diskussionen über die Lösung hinlänglich bekannter Probleme im deutschen Gesundheitswesen war.

Frisch im Amt, sah sich die neue politische Führung im Bundesministerium für Gesundheit schnell mit den Kernfragen der Versorgungslandschaft konfrontiert: Subjektiv empfundener Arztmangel trotz insgesamt gestiegener Arztzahlen bedingt durch eine sehr heterogene Verteilung ärztlicher Niederlassungen in der Fläche, nach wie vor mangelnde Integration der "Sektoren « Krankenhaus und Arztpraxis, Leerstand in jedem fünften Krankenhausbett, die demographische Entwicklung insgesamt. In ihren »Überlegungen zu einem Versorgungsgesetz « skizzierte die FDP im März 2011 eine Reihe von Maßnahmen, um den "großen Herausforderungen « im Kontext des demogra- 
phischen Wandels zu begegnen. Die Vorschläge umfassten eine "Landarztquote«, die Aufhebung der Residenzpflicht für Vertragsärzte, die Etablierung mobiler Arztstationen, den Ausbau der Delegation ärztlicher Leistungen an medizinische Assistenzberufe sowie eine verbesserte, flexiblere Bedarfsplanung. Als Anreiz für die Leistungserbringung in unterversorgten Gebieten waren darüber hinaus Modellversuche zur Prüfung von Formen der Kostenerstattung vorgeschlagen worden. Beim Abbau von Überversorgung wurde vollständig darauf gesetzt, dass die Kassenärztlichen Vereinigungen (KV) einen freiwilligen Verzicht auf eine Zulassung fördern können sollten. Die Aufhebung von Budget-Obergrenzen für das ambulante Operieren stellte den einzigen Vorschlag für einen »fairen wettbewerblichen Rahmen an der Schnittstelle von ambulant und stationär« dar. Die Anregungen zu einer Strukturreform der zahnärztlichen Vergütung beschränkten sich im Wesentlichen auf die Idee der Aufgabe der Budgetierung in diesem Bereich.

Auch die CDU/CSU-Fraktion im Deutschen Bundestag legte im Frühjahr 2011 unter der Überschrift »Das Angebot vom Bedarf des Patienten her gestalten « in 14 Punkten Vorschläge für eine Reform der medizinischen Versorgung in Deutschland vor. Den Autoren war offensichtlich bewusst, dass sie sich mit diesem Titel mutig aus der Deckung wagen - gleich in den ersten Sätzen wird darauf hingewiesen, dass Bedarfsgerechtigkeit ein eher subjektiver Begriff sei. Der allgemeinen Wahrnehmung einer »schlechteren Versorgungssituation« mit 383 Ärzten pro 100.000 Einwohnern im Jahr 2008 stünden 300 Ärzte pro 100.000 Einwohner 1990/91 gegenüber - damals wurde eine "Ärzteschwemme« diskutiert. Das Papier der Union zog den Schluss: »Vor dem Hintergrund dieser Zahlen kann jedenfalls bei globaler Betrachtung nicht von einem generellen Versorgungsproblem in Deutschland gesprochen werden.«. Da dennoch der von ärztlichen Verbänden proklamierte Mangel an 15.000 bis 20.000 zusätzlichen Niederlassungen in ländlichen Regionen Eingang in die Situationsbeschreibung fand, waren eine Reihe von Vorschlägen zur Überwindung von Versorgungsdefiziten aufgeführt worden. So sollten sektorübergreifende regionale Versorgungsausschüsse zur Versorgungsplanung eingerichtet werden, denen Vertreter der KVen, der Landesärztekammern, der Landeskrankenhausgesellschaft, der gesetzlichen Krankenkassen sowie des zuständigen Landesministeriums angehören. Grundlage der Planung sollten dabei nicht mehr die in den 1990er Jahren festgeschriebenen einheitlichen Vorgaben von Arzt-Einwohner-Relationen sein, sondern die Versorgungsbedarfe vielmehr auf der Basis bundesweit gültiger Kriterien aus den konkreten Notwendigkeiten der demographischen Entwicklung einer Region heraus kontinuierlich aktualisiert werden. Als denkbare Regelungen zum Abbau von Überversorgung und Fehlsteuerung wurden von der Union ein Verzicht auf Nachbesetzungen in überversorgten Gebieten sowie die Ermöglichung einer zeitlich befristeten Zulassung »in Ergänzung der lebenslangen Zulassung unter Berücksichtigung des Bestandsschutzes « ebenso wie eine Landarztquote und der Ausbau der Delegation ärztlicher Leistungen vorgeschlagen. Mit der Stärkung des Belegarztwesens und einer Verbesserung des Entlassmanagements sollte die Kooperation von niedergelassenen Ärzten und Krankenhäusern gestärkt werden. Als weitere Maßnahmen zur Förderung der
Attraktivität des Arztberufes wurden die Abschaffung der Richtgrößenprüfung für Heil- und Arzneimittel in der bisher bekannten Form sowie der Verzicht auf die Abstaffelung von Vergütungen in unterversorgten Regionen angeregt.

\section{Obgleich sich Motivation und Schwerpunktsetzung des GKV- VStG rhetorisch im Bereich der Schaffung einer adäquaten Versorgungsdichte verorten lassen, bildet die neuerliche Reform der vertragsärztlichen Vergütung einen besonderen Schwerpunkt.}

Obgleich sich Motivation und Schwerpunktsetzung des GKV-VStG rhetorisch im Bereich der Schaffung einer adäquaten Versorgungsdichte verorten lassen, bildet die neuerliche Reform der vertragsärztlichen Vergütung einen besonderen Schwerpunkt. Damit wird die Wertehierarchie des Koalitionsvertrags wieder hergestellt: Während bereits im Entstehen des schwarz-gelben Regierungsbündnisses die Ansicht artikuliert wurde, dass die honorarpolitischen Regelungen auf den Prüfstand gehören, stand die flächendeckende Versorgung im Oktober 2009 noch nicht breit auf der offiziellen Agenda.

\section{Honorarreform}

Der honorarpolitische Handlungsbedarf war das Ergebnis eines erheblichen Drucks aus der Ärzteschaft, die trotz historischer Rekordzuwächse bei der Gesamtvergütung eine Revision der Honorarreform forderte. Im Fokus der Unzufriedenheit stand dabei insbesondere die Systematik der Regelleistungsvolumina (RLV), die von vielen Ärzten als intransparent empfunden und als Ursache von bestehenden Verteilungsungerechtigkeiten angesehen wurde.

Im GKV-Finanzierungsgesetz (GKV-FinG) erfuhr die Überprüfung und Korrektur der Honorarreform mit dem neu eingefügten $\mathbb{\int} 87$ Abs. 9 SGB V erstmalig eine legislative Umsetzung, indem der Bewertungsausschuss mit der Erstellung eines Konzepts zur Konvergenz der Vergütungen beauftragt wurde. Die Koalition orientierte sich damals an einem zentralen Anliegen der Kassenärztlichen Bundesvereinigung (KBV), deren Vertreterversammlung in einer außerordentlichen Sitzung eine solche Angleichung der regionalen Gesamtvergütungen als mittelfristiges Ziel eines kassenärztlichen Honorarkonzepts im Mai 2010 beschlossen hatte.

In der Selbstverwaltung kam eine Einigung über ein Konvergenzkonzept nicht in der gesetzlichen Frist zustande. Dies lag allerdings nicht nur an den diametral entgegengesetzten Positionen der Bänke - gleichzeitig wurde die Konsensfindung dadurch erschwert, dass die Vorstellungen der KBV keine hinreichende Mehrheit unter den KVen fanden.

Der »Schwebezustand «, der durch den Auftrag an die Selbstverwaltung und die ausbleibende Einigung im Bewertungsausschuss Ärzte eingetreten war, schlug sich auch in den 
Eckpunktepapieren zum Versorgungsstrukturgesetz wieder, die den gesamten Themenbereich der Honorarreform nur rudimentär behandelten. Als Beispiel können hier die 14 Punkte der Unionsfraktion aus dem Januar 2011 dienen, in denen hauptsächlich auf den Prüfauftrag an die Selbstverwaltung verwiesen wurde und in dem sich eine Collage punktueller Regelungsabsichten offenbarte, die in Kontext, Detailtiefe und Substanz sehr heterogen waren. In den gemeinsamen Eckpunkten der Regierungsfraktion aus dem April war die Entscheidungsfindung zumindest so weit gediehen, dass die zukünftige Stoßrichtung in der Honorarverteilung vorgezeichnet war. Gleichzeitig machen diese Papiere deutlich, dass bis kurz vor dem Gesetzgebungsverfahren offenbar keine konzeptionelle Vorstellung über Handlungsbedarf und Lösungsalternativen in der vertragsärztlichen Vergütung bestand.

Mit Vorlage des Referentenentwurfs bestand dann Klarheit über die wesentlichen Regelungsbestandteile, die überwiegend bis zum Beschluss des Bundestages Bestand haben sollten. Neben der in den Eckpunkten bereits angestrebten Übertragung der Honorarverteilung in die Kompetenz der KVen wurde auch die Weiterentwicklung der Gesamtvergütung grundlegend neu geordnet.

Grundsätzlich können im Rahmen der Honorarreform vier wesentliche Komponenten unterschieden werden, die sich in den einzelnen Regelungsbestandteilen überlappen und Interdependenzen aufweisen:

- Honorarpolitische Maßnahmen, die im Zusammenhang mit dem eigentlichen Anlass der Gesetzgebung stehen und den Zweck verfolgen das Niederlassungsverhalten zu steuern

- Weitgehende Regionalisierung der vertragsärztlichen Vergütungsverhandlungen

- Stärkung der Kassenärztlichen Vereinigungen durch eine Monopolisierung der Honorarverteilung und der Informationen über die vertragsärztliche Vergütung

- Vorgaben zur Neuordnung des Einheitlichen Bewertungsmaßstabes, insbesondere hinsichtlich der Lockerung der Pauschalierung und der Berücksichtigung telemedizinischer Leistungen

Obgleich weitgehend Einigkeit in der Feststellung besteht, dass die Vergütung nur eine untergeordnete Bedeutung in der Niederlassungsentscheidung von werdenden Vertragsärzten ist, sind finanzielle Anreize ein wichtiges Instrument zur Steigerung der Motivation zur Praxiseröffnung in ländlichen oder ärmeren Regionen.

Aus diesem Grund hatte der Gesetzgeber bereits im Rahmen des GKV-Wettbewerbsstärkungsgesetzes ${ }^{1}$ eine Punktwertdifferenzierung in Abhängigkeit von der Versorgungssituation vorgesehen. Diese Regelung, die Abweichungen in beide Richtungen ermöglichte, wird nun durch eine reine Zuschlagsregelung ersetzt, die auf regionaler Ebene vereinbart werden kann.

Der Verhaltensökonomie verdanken wir allerdings die Erkenntnis, dass ein Individuum in der Regel den Verlust einer Sache, die sich bereits in seinem Besitz befindet, als subjektiv schwerwiegender wahrnimmt als deren Nichterwerb, obwohl objektiv kein Wertunterschied besteht. Überträgt man diese Beobachtung auf die Punktwertdifferenzierung, ist anzuneh- men, dass Punktwertabschläge eine höhere Anreiz- und damit auch Steuerungswirkung haben als Zuschläge. Es ist daher mit Hinblick auf die Steuerung des Niederlassungsverhaltens nicht schlüssig, ausgerechnet das wirksamere Instrument aus dem Gesetz zu streichen.

Vordergründig wird in der Gesetzbegründung mit einer Vereinfachung des Vergütungssystems argumentiert. Letztlich wird aber politische Opportunität den Ausschlag gegeben haben, die unumwunden in der axiomatischen Behauptung aus den Reihen der Koalitionsfraktionen zum Ausdruck gebracht wird, wonach eine gute Versorgung nur »mit« den, und nicht gegen die Ärzte organisiert werden könne.

Neben den Zuschlägen soll die Attraktivität der Tätigkeit in schlecht versorgten Regionen dadurch erhöht werden, dass in diesen Fällen eine Ausnahme von der Mengenabstaffelung gemacht wird und die betreffenden Ärzte eine Einzelleistungsvergütung erhalten. Die Idee einer solchen Regelung ist kein Novum, der Bewertungsausschuss hatte bereits einen entsprechenden Beschluss gefasst, der den Partnern der Gesamtverträge entsprechende Festlegungen in der Honorarverteilung ermöglichte. Neu ist hingegen der verpflichtende Charakter der Regelung, der womöglich Umsetzungsschwierigkeiten hervorrufen kann. Da Begriff und Definition der Unterversorgung derzeit Gegenstand von Beratungen im Gemeinsamen Bundesausschuss sind, ist noch nicht abschätzbar, in welchem Umfang Ärzte von dieser Regelung profitieren werden. Übersteigt diese Zahl allerdings eine kritische Masse, wird eine KV außerstande sein, die damit verbundenen Honorare aus den Fachgruppentöpfen zu bedienen, ohne Ärzte in nicht unterversorgten Regionen übermäßig zu belasten. Insbesondere bei den Hausärzten, deren Vergütungsvolumen noch strikt von den fachärztlichen Teilbudgets abgeschottet sind, kann es zu erheblichen Verwerfungen kommen, sollte der Unterversorgungsbegriff zu weit gefasst werden. In der Folge kämen auf die Krankenkassen Forderungen nach einem Vergütungsnachschuss zu, der auf Umwegen direkt in eine partielle Einzelleistungsvergütung führen würde.

Derartige Verknüpfungspunkte zwischen Honorarpolitik und Bedarfsplanung machen deutlich, dass beide Regelkreise nicht getrennt, sondern zunehmend als Einheit betrachtet werden müssen, um die gegenseitige Einflüsse berücksichtigen zu können.

\section{Die fast vollständige Verlagerung der Honorarverantwortung auf die Ebene der KV-Bezirke ist zugleich das vorläufige Ende jedes Strebens nach bundesweit einheitlichen Vergütungen.}

Die fast vollständige Verlagerung der Honorarverantwortung auf die Ebene der KV-Bezirke ist zugleich das vorläufige

\footnotetext{
1 Gesetz zur Stärkung des Wettbewerbs in der gesetzlichen Krankenversicherung (GKV-Wettbewerbsstärkungsgesetz, GKV-WSG), 26.03.2007
} 
Ende jedes Strebens nach bundesweit einheitlichen Vergütungen. Den Partnern der Gesamtverträge werden die Spielräume eröffnet, nahezu alle Vergütungssachverhalte zu verhandeln, deren Regelung früher dem Bewertungsausschuss oblag.

Ein auffälliges Novum ist die Ermittlung der morbiditätsbedingten Steigerungsrate der Gesamtvergütung, die sich nicht mehr als Resultat eines statistischen Rechengangs automatisch ergeben, sondern Ergebnis eines Verhandlungsprozesses sein soll. Dafür werden gleichwohl Leitplanken eingezogen, indem die Partner der Gesamtverträge jeweils eine demographische und eine diagnosebezogene Veränderungsrate durch den Bewertungsausschuss mitgeteilt bekommen und eine regionale Gewichtung treffen sollen.

Damit soll den regional unterschiedlich ausgeprägten Kodiereffekten Rechnung getragen werden, die sich bereits mit der Ankündigung automatisch ergeben, dass die Dokumentation von Diagnosen vergütungsrelevant ist. Hier wird auch die Abschaffung der Ambulanten Kodierrichtlinien eine Rolle spielen, die eigens für eine bundesweit einheitliche, regelgebundene Diagnosendokumentation erstellt worden waren: Eine zurückhaltende Gewichtung diagnosebezogener Steigerungsraten ist erforderlich.

Aber selbst der berechtigte Zweifel an der Aussagekraft derartiger Veränderungsraten wird die Krankenkassen in den kommenden Verhandlungen nicht davor bewahren, mit hohen Forderungen der Ärzteseite konfrontiert zu werden. Die KVen stehen nach der Realisierung der von ihnen geforderten Regionalisierung unter hohem Erfolgsdruck und werden die Vielzahl der zu verhandelnden Sachverhalte nutzen, um deutliche Vergütungssteigerungen zu begründen.

Adressat der umfassenden Kompetenzschiebungen sind in erster Linie die Kassenärztlichen Vereinigungen. Neben der Rückeroberung der Vergütungshoheit erhalten sie zukünftig die alleinige - allerdings an Vorgaben der KBV geknüpfte - Entscheidungsgewalt über die Honorarverteilung, die lediglich nur in einzelnen gesetzlichen Festlegungen ihre Schranken erfährt.

Die KVen erhalten damit eine Befugnis zurück, die ihnen mit dem Gesundheitsmodernisierungsgesetz insofern genommen wurde, als dass Einvernehmen mit den Krankenkassen herzustellen war. Der Gesetzgeber zieht damit die Konsequenz aus der in der Ärzteschaft weit verbreiteten Unzufriedenheit mit der RLV-Systematik. Diese Ermächtigung wird mit dem Etikett der Flexibilisierung versehen, Ziel und Zweck bleiben aber offen. Damit wird den KVen die Carte blanche erteilt, unabhängig von Versorgungsgesichtspunkten die Ressourcen zu steuern.

Dabei ist die Honorarverteilung in hohem Maße versorgungsrelevant. Die Entscheidung über die Allokation der Ressourcen hat Einfluss auf Art und Umfang der Leistungserbringung.

Während die Ärzte als Individuen in ihrer Mehrzahl ein ethisches Interesse an einer guten Versorgung ihrer Versicherten haben, trifft dies für die KVen als Institution weniger zu. Diese sind bestenfalls indifferent, wenn Kosten und Nutzen einer guten Versorgung für sie externe Effekte sind. Rational besteht allerdings eher ein Interesse an einer suboptimalen Versorgung, da zum einen die Inanspruchnahme anderer Leistungssektoren die eigenen Budgets entlasten kann und zum anderen Versorgungsmängel Ansatzpunkte für Sonder- verträge mit Krankenkassen darstellen, mithilfe derer die KVen zusätzliche Vergütungen generieren können. Es ist also für KVen durchaus rational, eben nicht im Interesse einer gu-

\section{Während die Ärzte als Individuen in ihrer Mehrzahl ein ethisches Interesse an einer guten Versorgung ihrer Versicherten haben, trifft dies für die KVen als Institution weniger}

zu.

ten Versorgung die Honorare zu verteilen. Vielmehr werden innerärztliche Proporz- und Besitzstandsgesichtspunkte bei der Verteilung die entscheidende Rolle spielen.

Vor diesem Hintergrund ist auch Skepsis im Hinblick auf die Möglichkeit von Sonderbudgets für Arztnetze angezeigt. Im Rahmen ihrer Honorarverteilungsautonomie sind die Kassenärztlichen Vereinigungen ausdrücklich ermächtigt, Arztnetze besonders zu berücksichtigen, wobei Kriterien des Bewertungsausschusses zu beachten sind. Inwieweit sich Hoffnungen der betreffenden Leistungserbringer, nun auch im Kollektivvertrag eine bessere Förderung und Autonomie zu erhalten, realisieren werden, ist ebenso offen, wie Befürchtungen der Krankenkassen, dass die selektivvertraglichen Beziehungen zu Praxisverbünden durch die KVen unterminiert werden könnten. Da nicht damit zu rechnen ist, dass die KVen den Arztnetzen mehr Mittel zur Verfügung stellen, als den darin zusammengeschlossenen Ärzte sonst individuell zugestanden worden ist, ist es allerdings fraglich, ob den Arztnetzen mit dieser reinen Delegation der Honorarverteilungsfunktion gedient ist. Der Vorteil direkter Vertragsmodelle, in denen durch eine Optimierung der Patientenversorgung zusätzliche Mittel für die Vergütung der Arztnetze generiert werden können, besteht in einer rein »internen « Regelung durch die KVen nicht.

In der Gesamtschau hat die Koalition eine tiefgreifende Kompetenzrochade in der vertragsärztlichen Vergütung auf den Weg gebracht, deren Zielrichtung jedoch mit Ausnahme derjenigen Maßnahmen, die unmittelbar der Niederlassungssteuerung dienen sollen, weitgehend im Unklaren bleibt. Die Schlagworte der Flexibilisierung und Regionalisierung sind eine Wegbeschreibung, wohin dieser Weg nach Ansicht der Koalition führen soll, kann aus dem Gesetzentwurf nicht erschlossen werden.

Auffällig ist jedoch die Rolle rückwärts in die Welt des Kollektivvertrags, die mit den letzten Gesundheitsreformen eigentlich schrittweise überwunden werden sollte. Die KVen gewinnen mit ihrer nun erheblich höher bewerteten Rolle in der vertragsärztlichen Vergütung auch als Vertragspartner für die Krankenkassen an Bedeutung. Die Honorarverteilung wird ein geschlossenes System, dass einer äußeren Nachvollziehbarkeit oder gar Kontrolle unzugänglich ist. Auf diese Weise bedient die Honorarreform in erster Linie Interessenlagen des KV-Systems, deren wirkungsvolle Kommunikation und die konzeptionelle Notlage, in der sich die Koalition im Frühsommer 2011 befand, offenbar im vertragsärztlichen Sinne harmonierten. 
Es zeigt sich, dass interessenpolitischer Einflussnahme dann in besonderem Maße der Boden bereitet ist, wenn sich ein abstrakter Handlungsbedarf, das diffuse Empfinden, dass etwas womöglich dringend getan werden müsse, mit ordnungspolitischer und konzeptioneller Orientierungslosigkeit vereint. Selbst der ehrenhafteste Widerwille gegen interessengeleitete Politikberatung weicht in jenen Momenten tiefster Dankbarkeit für das inhaltliche Angebot, dass zur Füllung dieses Vakuums geeignet ist.

Allerdings ist mit diesen großen Zugeständnis an die organisierte Ärzteschaft gleichzeitig die Erwartung verbunden, dass von nun an, da es die KVen selbst in der Hand haben, sich der politische Druck künftig nach innen richtet. Den von der Wanderung der Verantwortung ausgetretenen Pfaden, so das politische Kalkül, werden alsbald die Schuldzuweisungen folgen können, wenn aus ärztlichem Munde weiterhin die Unzufriedenheit in die Öffentlichkeit findet - Verantwortungs- und Gestaltungsverzicht als vorbeugende Exkulpierungsmaßnahme.

Dabei ist ein Scheitern der KVen in ihrer Aufgabe, ihre Kompetenzfülle zur Befriedung der Ärzteschaft auszuüben, offenbar einkalkuliert, was sich an der Ermächtigung der $\mathrm{KBV}$, zur Honorarverteilung verbindlichen Vorgaben zu machen, ablesen lässt. Von dieser Option wird die KBV zu Beginn möglicherweise nur sehr zurückhaltend Gebrauch machen, kann diese bei Bedarf aber jederzeit ausüben. Eine Zentralisierung der Honorarverteilung ist so ohne Gesetzesänderung flexibel möglich.

Bei der sorgfältigen Austarierung institutioneller Interessen bleibt für die Versorgung nur eine untergeordnete Bedeutung. Dieser Mangel an Versorgungsorientierung in der Honorarpolitik ist bemerkenswert, wenn man den legislativen Kontext berücksichtigt, in den die Honorarreform eingebettet ist.

Dabei bleibt die zentrale Herausforderung der vertragsärztlichen Vergütung weiterhin unberührt: Die Honorarungerechtigkeit zwischen und innerhalb von Arztgruppen. Mit der Wahl der Fachrichtung trifft ein angehender Arzt gleichzeitig die Entscheidung über sein realistisch erzielbares Einkommen. Nach Angaben des Statistischen Bundesamts kann beispielsweise ein Mediziner, der sich für die Allgemeinmedizin entscheidet, um Hausarzt zu werden, lediglich ca. $50 \%$ des Einkommens (nach Abzug aller Praxiskosten) eines fachärztlichen Internisten erwarten.

Diese Spreizung der Einkommen hat Konsequenzen für die Versorgung. Zum einen ist damit zu rechnen, dass junge Mediziner sich für Fachrichtungen entscheiden, in denen neben dem Prestige auch höhere Verdienstmöglichkeiten zu erwarten sind. Zum anderen weichen Vertragsärzte auf lukrative Leistungen innerhalb, aber auch außerhalb des vertragsärztlichen Leistungsgeschehens aus. In dem einen Fall verengen sie das Leistungsangebot für die gesetzlich Versicherten, in dem anderen wird das gesamte Angebot verknappt.

Derartigen Fehlentwicklungen wird mit den im GKVVersorgungsstrukturgesetz enthaltenen Instrumenten nicht annährend begegnet. Es stellt sich die Frage, inwieweit die Bemühungen, den Hausarztberuf attraktiver zu machen, nicht leerlaufen, wenn auch weiterhin die Entscheidung für den Hausarztberuf mit der Halbierung des zu erwartenden Einkommens einhergeht. Die Einkommensunterschiede sind derart deutlich, dass Punktwertzuschläge nicht ausreichen bzw. in dafür notwendiger Höhe nicht finanzierbar wären - schließlich bedeutete dies eine Anhebung des Vergütungsvolumens, und in entsprechender Höhe auch der Leistungsbewertungen, der Hausärzte, aber auch anderer Fachgruppen, um ca. $50 \%$.

\section{Laufen die Bemühungen, den Hausarztberuf attraktiver zu machen, nicht leer, wenn weiterhin die Entscheidung für den Hausarztberuf mit der Halbierung des zu erwartenden Einkommens einhergeht.}

Daher kann der Weg nur über eine schrittweise Angleichung der Arzteinkommen führen, im Sinne einer Chancengleichheit, dass für jeden Arzt bei gegebener Leistung und Arbeitszeit das gleiche Arzteinkommen erzielbar ist. Die Neukalkulation des einheitlichen Bewertungsmaßstabs für ärztliche Leistungen (EBM), eine hinreichende Bedingung für das Erreichen dieses Ziels, ist Gegenstand laufender Beratungen. Unabhängig davon, ob eine faire und sachgerechte Neubewertung der EBM-Leistungen erreicht wird, ist darüber hinaus die Anpassung der Fachspezifischen Teilbudgets in der Honorarverteilung notwendig, um der Leistung auch wirklich Geld folgen lassen zu können. Durch das Versorgungsstrukturgesetz obläge es nun den KVen alleine, um die erforderlichen Umverteilungsmaßnahmen zu unternehmen. Erfahrungsgemäß besteht hier eine schlechte Prognose. Die KBV resümiert zum GKV-VStG jedenfalls prompt: »Die Politik verlässt damit endlich den Weg der Kostendämpfung im Gesundheitswesen.«

\section{Spezialfachärztliche Versorgung}

Um einheitliche Rahmenbedingungen und Vergütungen an der Sektorengrenze zu schaffen, forderte die Union in ihrem 14-Punkte-Papier im März 2011 einheitliche Qualitätskriterien für die Leistungserbringung sowie die Einführung einer einheitlichen pauschalen Vergütung für ambulante ärztliche Leistungen. Über die Zulassung von Leistungserbringern sollten die sektorübergreifenden regionalen Versorgungsausschüsse befinden.

Der GKV-Spitzenverband hatte in diesem Zusammenhang angeregt, die ambulante Versorgung im Krankenhaus grundsätzlich neu zu ordnen und für die Vielzahl verschiedener gesetzlicher Regelungen zur Einbindung der Krankenhäuser in einem einheitlichen rechtlichen Rahmen neu zu fassen. Bislang sind teilstationäre Behandlung ( $\int 39$ SGB V), vorund nachstationäre Behandlung ( $\mathbb{S} 115$ SGB V), ambulantes

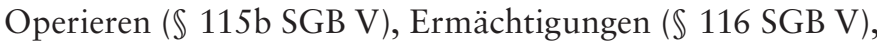
Disease Management-Programme ( $\int 116$ b Abs. 1 SGB V), die Behandlung von seltenen Krankheiten und Krankheiten mit besonderem Verlauf ( $\mathbb{1} 116$ b Abs. 2 - 4 SGB V), Hochschulambulanzen ( $\$ 117$ SGB V), Psychiatrische Institutsambulanzen (\$118 SGB V), Sozialpädiatrische Zentren ( $\$ 119$ SGB V), 
Pädiatrische Spezialambulanzen ( $\mathbb{1 2 0}$ Abs. 1a SGB V) sowie die Integrierte Versorgung ( $\mathbb{S} 140 \mathrm{a}$ ff. SGB V) mit unterschiedlichen Zulassungsbedingungen sowie verschiedenen Vergütungswegen und heterogenen Vorgaben zur Qualitätssicherung geregelt.

Der AOK-Bundesverband schlug vor, "Zulassungen « und »Ermächtigungen« zur ambulanten Versorgung als Zulassungen zusammenzufassen und die Zulassungsausschüsse in den Ländern künftig grundsätzlich sektorübergreifend aufzustellen. Leistungserbringer aus dem Bereich der fachärztlichen Versorgung sollten bei dem sektorübergreifenden Zulassungsausschuss die Zulassung beantragen, die sowohl für Ärzte, die die allgemeinen Zulassungsbedingungen gem. $\mathbb{9} 95 \mathrm{Abs.} 2$ SGB V erfüllen, wie auch für zugelassene Vertragsärzte und Krankenhäuser gelten kann. Der Versorgungsauftrag ließe sich so auf bestimmte Leistungsbereiche, -inhalte bzw. -volumina begrenzen. Idealerweise sollten Zulassungen auch zeitlich befristet ausgesprochen werden können.

Mit dem GKV-VStG wird der $\mathbb{S} 116 \mathrm{~b}$ SGB V nun nach monatelangem Ringen zwischen Bund und Ländern unter dem Titel »Spezialfachärztliche Versorgung « neu gefasst und damit ein neuer, »sektorenverbindender « Versorgungsbereich unter Einbeziehung der Vertragsärzte eingeführt. Gegenstand sind Diagnostik und Therapie »komplexer, schwer therapierbarer Krankheiten, die je eine spezielle Qualifikation, interdisziplinäre Zusammenarbeit und oder besondere Ausstattungen erfordern«. Dazu zählen schwere Verlaufsformen von Erkrankungen mit besonderen Krankheitsverläufen, seltene Erkrankungen ${ }^{2}$, Erkrankungen mit geringen Fallzahlen sowie hoch spezialisierte Leistungen. Das Gesetz sieht eine konkrete Liste von Indikationen vor, die im Wesentlichen den seltenen Krankheiten und Krankheiten mit besonderem Verlauf gemäß der bisherigen Fassung des $\mathbb{1 1 6 b}$ SGB V entsprechen. Der Gemeinsame Bundesausschuss (G-BA) kann den Katalog der Indikationen auf Antrag einer seiner Trägerorganisationen, der Patientenvertreter oder eines Unparteiischen ergänzen. Das Prinzip des Verbotsvorbehalts für die Anwendung einzelner Untersuchungs- und Behandlungsmethoden des stationären Sektors gilt und wird damit nun auch auf einen Teil der ambulanten Versorgung ausgedehnt.

Bis zum 31.12.2012 soll der G-BA in einer Richtlinie "Näheres" zur Leistungserbringung in der ambulanten spezialfachärztlichen Versorgung regeln. Dabei bestimmt er den Behandlungsumfang inkl. der Anforderungen an Leistungserbringung und Qualitätssicherung. Zur Behandlung schwerer Verlaufsformen von Erkrankungen mit besonderen Verläufen ist die Überweisung durch einen Vertragsarzt Voraussetzung für die Inanspruchnahme - auch hier regelt der G-BA Näheres. Für seltene Erkrankungen sowie hoch spezialisierte Leistungen legt er fest, in welchen Fällen eine Überweisung durch den behandelnden Arzt vorausgesetzt wird. Zudem kann der G-BA Entscheidungshilfen in Form von Empfehlungen vorgeben, in welchen Fallkonstellationen von einem besonderen Krankheitsverlauf auszugehen ist. Für schwere Verlaufsformen onkologischer Erkrankungen hat er Regelungen zu Vereinbarungen für die Förderung der Kooperation zwischen den beteiligten Leistungserbringern zu erarbeiten; für schwere Verlaufsformen von Erkrankungen mit besonderen Verläufen kann er Vorgaben für derartige Vereinbarungen vorsehen.
Die Leistungen der ambulanten spezialfachärztlichen Versorgung werden direkt zwischen Leistungserbringer und Krankenkasse abgerechnet; Vertragsärzte können KVen entgeltlich mit der Abrechnung beauftragen. GKV-Spitzenverband, Deutsche Krankenhausgesellschaft und KBV sollen eine einheitliche Kalkulationssystematik und diagnosebezogene Gebührenordnungspositionen in Euro vereinbaren, deren Kalkulation vom EBM ausgeht. Bis zur Vereinbarung dieser Gebührenordnung wird der EBM zur Abrechnung genutzt, der zu diesem Zweck vom Bewertungsausschuss Ärzte so anzupassen ist, dass die Leistungen der spezialfachärztlichen Versorgung »angemessen« berücksichtigt sind und zur Abrechnung genutzt werden können. Die Prüfung von Abrechnung, wirtschaftlicher Leistungserbringung und Qualität obliegt den Krankenkassen, die damit auch ihren Medizinischen Dienst beauftragen können. Eine Bereinigung der morbiditätsbedingten Gesamtvergütung um die Leistungen der spezialärztlichen Versorgung aus dem über die Grundversorgung hinausgehenden fachärztlichen Vergütungsanteil ist vorgesehen.

Da der EBM im Kern leistungsbezogen gestaltet ist, bei der Neukalkulation von Pauschalen für Fälle und Behandlungsepisoden aber Leistungen auch differenzierend zu aggregieren sind, wäre für die Gestaltung der neuen Pauschalen ein Rückgriff auf Erfahrungen aus der DRG-Kalkulation sicherlich zielführender. Damit hätte auch die Möglichkeit bestanden, die Pauschalen derart zu gestalten, dass sie als Nukleus für ein sektorunabhängig gestaltetes Vergütungssystem dienen können.

Für die Zulassung der Leistungserbringer gilt eine generelle Erlaubnis mit Verbotsvorbehalt: Sie sind bei Vorliegen aller vom G-BA formulierten Anforderungen und fehlendem Widerspruch nach Ablauf einer Frist von zwei Monaten nach Eingang der jeweiligen Anzeige bei der zuständigen Landesbehörde zur Teilnahme berechtigt. Da der G-BA sächliche und personelle Anforderungen definieren wird bleibt offen, warum das Gesetz bei der Zulassung durch die Landesbehörden auf eine zwingende Prüfung des Vorliegens der entsprechenden Voraussetzungen verzichtet.

Der Referentenentwurf des GKV-VStG sah vor, auch das ambulante Operieren in die spezialfachärztliche Versorgung zu überführen; der Katalog der Erkrankungen mit besonderen Verläufen war initial noch nicht auf schwere Verlaufsformen beschränkt. Vor diesem Hintergrund wurde intensiv die Notwendigkeit von Maßnahmen zur Mengensteuerung diskutiert. Gerade bei den Leistungen des ambulanten Operierens sowie bei besonderen Krankheitsverläufen ist von einer hohen Bandbreite der Indikationsstellung auszugehen: Die Mengenentwicklung bei der operativen Behandlung der Katarakt in den vergangenen Jahren ist dafür ein eindrucksvolles Beispiel. Das ambulante Operieren wurde letztlich nicht Gegenstand des Leistungskatalogs des $\mathbb{1} 116 \mathrm{~b}$ SGB V GKV-VStG; die Behandlung schwerer Verlaufsformen onkologischer Erkrankungen oder der Herzinsuffizienz bergen jedoch nach wie vor Mengenrisiken, die auch durch den kurz vor dem Beschluss des Gesetzes noch aufgenommenen Überweisungsvorbehalt nicht eliminiert werden.

2 Definition »Seltene Erkrankung« in der EU: Prävalenz von 1/2.000 
Die Konstruktion der spezialfachärztlichen Versorgung unter Verzicht auf eine vertragliche Grundlage stellt gerade vor diesem Hintergrund ein besonderes Problem dar. In einem Versorgungsbereich ohne vertragliche Beziehung zwischen Leistungserbringern und Krankenkassen fehlt den Kostenträgern eine zentrale Möglichkeit, die Versorgungslandschaft im Sinne ihrer Versicherten mitgestalten zu können; der Versorgungsmarkt wird so wesentlich stärker von den Anbietern geprägt und tendiert zu intensiverer Leistungsinduktion. Im Kontext überproportional steigender Arztzahlen und einer beispiellosen Zahl von Konsultationen ${ }^{3}$ ein fragwürdiger An-

\section{Die Gestaltung der spezial- fachärztlichen Versorgung nach dem GKV-VStG oktroyiert den Kostenträgern, sämtliche Leistungsvolumina zu akzeptieren und zu vergüten}

satz. Die Gestaltung der spezialfachärztlichen Versorgung nach dem GKV-VStG oktroyiert den Kostenträgern, sämtliche Leistungsvolumina zu akzeptieren und zu vergüten, die in diesem Bereich von Praxen und Krankenhäusern angeboten werden. Einen Denkanstoss erhalten hier jene, die Schwierigkeiten hatten, sich etwas Konkretes hinter dem oft etwas wolkig bemühten Terminus von der »Verstaatlichung des Gesundheitswesens" vorzustellen. Die Gelegenheiten, vertragslose, und damit konstitutive Merkmale von Märkten ignorierende Ordnungsvorgaben mit »echten « Liberalen zu diskutieren, werden offensichtlich immer seltener.

Das Vorliegen der vom G-BA zu formulierenden Anforderungen an die Leistungserbringung stellt künftig die einzige Hürde dar, um im Bereich der spezialfachärztlichen Versorgung tätig zu werden. Überlegungen zu einer verpflichtenden Befristung der Zulassungen verschloss sich der Gesetzgeber.

\section{Neuregelungen zur Bedarfsplanung}

Eines der wesentlichen Probleme der deutschen Versorgungslandschaft bleibt die Verteilung der ärztlichen Niederlassungen in der Fläche. Zur Verbesserung der Versorgungssituation auf dem Land schnürte die Regierungskoalition mit dem im Vorfeld auch als "Landärztegesetz « bezeichneten Gesetzgebungsvorhaben zum GKV-VStG ein weiteres Maßnahmenbündel. Die Niederlassung für Ärzte in infrastrukturschwachen Regionen soll attraktiver werden. Dabei geht es der Regierungskoalition um mehr als nur um Landärzte. »Es geht um die Versorgung in der Fläche, um die Freiberuflichkeit«, führt der Bundesgesundheitsminister aus. »Wir haben immer mehr Ärztinnen und eine andere Einstellungen junger Mediziner zum Arztberuf. Stichworte sind hier Vereinbarkeit von Familie und Beruf und die Dezentralisierung von Entscheidungen. Das charakterisiert einen grundlegenden Richtungswechsel in der Gesundheitspolitik. Vor allem wollen wir jungen Medizinern eine verlässliche Perspektive geben. $\aleph^{4}$ Reichen die Anreize also aus, um niederlassungswillige junge Ärzte in den ländlichen Raum zu locken und dort langfristig zu binden?

Schon im Vorfeld des Gesetzgebungsverfahrens zeigte sich, in welche Richtung die Debatte steuerte. Orientierte sich die Bedarfsplanung und Zulassungspolitik bislang noch an der in den 90er Jahren postulierten Ärzteschwemme, vergrößerten sich die Lücken in der Fläche trotz eines Anstiegs der Arztzahlen seit 1990 um mehr als 50 \%. Das Ergebnis der Verwaltung und Verwehrung von Niederlassungsrechten hatte keineswegs eine bedarfsgerechte Verteilung von Ärzten gefördert. Die regional sehr unterschiedlichen Einschätzungen von spezifischen Versorgungsbedürfnissen und eine ebenso individuell geprägte Zulassungspraxis führten in der Vergangenheit zu einer Spreizung der ambulanten Versorgungsdichte in einigen Fachgruppen, die allein durch Unterschiede in der Morbidität nicht zu erklären ist. Die Krankenkassen wiesen unermüdlich darauf hin, dass es nicht an Ärzten mangele, sondern deren Verteilung in der Fläche gescheitert und die Behebung dieser Versorgungsdisparitäten für den Erfolg einer zukünftigen Planung der Versorgung entscheidend sei. Die Ärztevertreter wiesen ebenso beharrlich auf den drohenden Ärztemangel hin. Dabei war allen Beteiligten klar, dass für einen Arzt, der sich im ländlichen Raum ein Leben lang verpflichten soll, nicht allein wirtschaftliche Anreize entscheidend sind. Für die Standortwahl geben weitere Bedürfnisse den Ausschlag. Ging es zunächst darum, Versorgungsbedarf sachgerecht zu ermitteln und geeignete Versorgungskonzepte zu entwickeln, ging es schließlich darum, die Versorgungsbedürfnisse von Ärzten in weniger attraktiven Regionen zu erfüllen.

\section{Allen war klar, dass für einen Arzt, der sich im ländlichen Raum niederlässt, nicht allein wirtschaftliche Anreize entscheidend sind.}

Der Gesetzgeber hat mit dem Versorgungsstrukturgesetz (VStG) in erster Linie die ambulante Bedarfsplanung für die vertragsärztliche Versorgung neu geregelt. Die grundlegende Systematik der Bedarfsplanung bleibt bestehen und die Richtlinienkompetenz des G-BA erhalten. Darüber hinaus ist keine überzeugende Absicht zu erkennen, die sich auf eine langfristige Versorgungsplanung richtet und den ambulanten wie auch den stationären Sektor gleichermaßen in die Pflicht nimmt. Das Gesetz lockert die Zulassungs- und Niederlassungsbedingungen und überlässt es mit wenigen einschränkenden Vorgaben der Selbstverwaltung, neue Parameter für die Gestaltung von Versorgungsräumen und Versorgungsgraden zu finden. Insbesondere aber stärkt es die Aufsichts- und Beteilungsrechte der Länder als subsidiäre Sicherstellungsträger, sowohl auf Bundes- wie auch auf Landesebene.

Dass die bestehenden Planungsräume zu groß sind, um

\footnotetext{
3 Barmer GEK Arztreport 2010: Im Mittel 18,1 Arztbesuche in Deutschland im Jahr 2008

4 Bahr: Klientelismus bei der FDP ist schlichter Unsinn. Ärzte Zeitung, 22.12.2011, http://www.aerztezeitung.de/politik_gesellschaft/gp_specials/jahresendausgabe-2011/article/683793/bahr-klientelismus-fdpschlichter-unsinn.html
} 
die Verteilung in der Fläche zu er-möglichen, steht außer Frage. Die Richtlinie des G-BA, mit der die Bedarfsplanung bundes-weit die Vorgaben für die ambulante vertragsärztliche Versorgung normiert, soll deshalb bis Ende 2012 überarbeitet werden. Die Räume erhalten einen neuen Zuschnitt, der flexibler und auch auf regionaler Ebene gestaltbar ist. Der Spezialisierungsgrad der Arztgruppen und die Häufigkeit der Inanspruchnahme legen mindestens drei Typen von Bezugsräumen nahe, um Zulassungen zu verwalten und die Arztdichte zu regulieren. Maßgeblich für deren Neugestaltung ist nach dem Willen des Gesetzgebers die wohnortnahe Versorgung. Wegen der unterschiedlichen Bedeutung der Wohnortnähe ist für verschiedene ärztliche Angebote eine Differenzierung nach Arztgruppen möglich. ${ }^{5}$ So werden jeweils ein Raumtyp für eine primär-ärztliche, eine fachärztliche und eine spezialärztliche Versorgung favorisiert. Die Unterscheidung der bislang zehn Regionstypen mit insgesamt 395 Planungsräumen als Bezugsgröße für die Zulassung von Ärzten aus 14 Arztgruppen wird aufgegeben. Die Planungsbereiche müssen nicht mehr den Stadt- und Landkreisen entsprechen. Da der Gesetzgeber damit ein vergleichsweise rechtssicheres Instrument etabliert hatte, das dann seine volle Wirkung entfaltete, wenn weitere Zulassungen nicht erwünscht waren, bleibt abzuwarten, ob die neue Bedarfsplanungs-Richtlinie tatsächlich Raumbezüge jenseits von Kreis oder Gemeindegrenzen festlegt.

Mithin wird die bestehende Bedarfsplanung systematisch weiterentwickelt und nur in einigen Details verändert und erweitert. Zentrales Anliegen ist es jedoch zunächst, die Voraussetzungen für eine flächendeckende Versorgung zu schaffen. Das kann nicht gelingen, wenn nicht dort, wo nach heutiger Bedarfsplanung eine weit überdurchschnittliche Versorgungsdichte besteht, auf die Nachbesetzung von Praxissitzen verzichtet wird. Das VStG eröffnet den Zulassungsausschüssen diesen Weg als gemeinsame Entscheidung, die Ärzte und Krankenkassen zu treffen haben. Außerdem können neue Zulassungen in Planungsbereichen ab einem Versorgungsgrad von $100 \%$ befristet werden, sofern sie nicht bereits gesperrt sind. Sollten diese Regelungen von den Beteiligten nicht in einem gemeinsamen Verständnis der flächendeckenden Sicherstellung genutzt werden, wird die gewachsene ungleiche Verteilung konserviert und ein zentrales Anliegen des VStG nicht beachtet.

Nachdem die KBV Ende 2009 freimütig eingeräumt hatte, die ambulante Sicherstellung nicht mehr ohne Beteiligung des stationären Sektors gewährleisten zu können, schlug sie eine sektorenübergreifende Versorgungsplanung durch einen regional tätigen öffentlich-rechtlichen Zweckverband vor. Die Länder bekundeten in den folgenden beiden Jahren 2010 und 2011 ihre Pflicht, der Gesamtverantwortung für die gesundheitliche Daseinsfürsorge stärker Rechnung tragen zu wollen und rangen um geeignete Maßnahmen. Im Verlauf der Beratungen eröffnete sich ein Einigungskorridor, um die Einflussmöglichkeiten der zuständigen Landes- und Verwaltungsbehörden auf die ambulante Bedarfsplanung mit dem VStG ausgesprochen zu stärken. Die Vertreter der für die Sozialversicherung zuständigen obersten Landesbehörden wirken nun beratend an den Sitzungen der Landesausschüsse mit und haben ein Recht auf Anwesenheit bei der Beschlussfassung. Zudem erhalten sie Mitberatungsrechte im für die Richtlinien zuständigen G-BA und auch hier dürfen sie bei der
Beschlussfassung anwesend sein. Aufsicht über die Landesausschüsse führen die für die Sozialversicherung zuständigen obersten Verwaltungsbehörden der Länder.

Die Länder hielten es für erforderlich, dass die für die Bedarfsplanung in der ambulanten und stationären Versorgung zuständigen Gremien durch ein weiteres sektorübergreifendes Gremium ergänzt werden. ${ }^{6}$ Der Bund stand der Schaffung eines solchen Gremiums zwar ablehnend gegenüber, schrieb aber das gemeinsame Landesgremium mit dem $\$ 90$ a SGB V ins Gesetz. In der nun gewählten Fassung bleibt vieles unverbindlich. Das gemeinsame Landesgremium kann optional von den Ländern eingerichtet und ausgestaltet werden. Die Anzahl der Mitglieder ist frei und setzt sich aus Vertretern des Landes, der KVen, der Krankenkassen, der Landeskrankenhausgesellschaften und weiteren nicht näher bestimmten Beteiligten zusammen. Das gemeinsame Landesgremium berät zwar sektorübergreifende Versorgungsfragen, kann aber lediglich Empfehlungen abgeben, Stellung zu Entscheidungen der Landesausschüsse nehmen und Fragen im Zusammenhang mit der spezialärztlichen Versorgung nach $\$ 116 \mathrm{~b}$ SGB V beraten. Es ist völlig offen, in welcher Weise die Beratungsergebnisse Wirkung entfalten und ob auch die Krankenhausplanung die Empfehlungen des Gremiums zur Kenntnis nimmt.

\section{Die Länder hielten es für erforderlich, dass die für die Bedarfsplanung in der ambulanten und stationären Versorgung zuständigen Gremien durch ein weiteres sektorübergreifendes Gremium ergänzt werden.}

Die überarbeitete Bedarfsplanungs-Richtlinie, welche am 01.01.2013 in der Neufassung in Kraft treten soll, wird sich auf wenige Erweiterungen beschränken müssen, mit denen auch stationäre Einrichtungen in eine sektorübergreifende Betrachtung der ambulanten Leistungserbringung einbezogen werden. Tatsächlich findet diese Versorgung an den Sektorengrenzen schon lange statt. Mit zahlreichen Sonderverträgen, die der Gesetzgeber sukzessive mit der Absicht vorgeschrieben hatte, spezifische Versorgungsbedürfnisse zu erfüllen, entlasten bereits Krankenhäuser die Vertragsärzte. Dazu zählen z. B. die Psychiatrischen Institutsambulanzen, die einen Beitrag zur Versorgung psychisch kranker Menschen leisten und als Institut entweder je nach Typ pauschal oder durch die Zulassungsausschüsse ermächtigt werden. Daneben erbringen unter anderen sozialpädiatrische Zentren, Hochschulambulanzen und individuell ermächtigte Ärzte definierte ambulante Leistungen in stationären Einrichtun-

\footnotetext{
5 Kabinettsentwurf Gesetz zur Verbesserung der Versorgungsstrukturen in der gesetzlichen Krankenversicherung. Gesetzesbegründung, S. 119

6 Kommission zur Sicherstellung der ärztlichen Versorgung in Deutschland: Ergebnisse der Klausurtagung vom 16. und 17.02. in Erkner zur Vorbereitung des Treffens der Staatssekretärinnen und Staatssekretäre am 29.03.2011
} 
gen. Der kontinuierliche Anstieg der dort behandelten Patienten und die häufig zu beobachtende Zuweisungen durch die Primärversorger (Haus- und Kinderärzte) werden gerne mit einer veränderten Morbidität und mit Versorgungsengpässen bei niedergelassenen Ärzten erklärt. Es ist durchaus zu begrüßen, dass ermächtigte Institute und Ärzte zukünftig in die Berechnung der Versorgungsgrade einbezogen werden. Jedoch können sie zur Sperrung von Planungsbereichen für eine Arztgruppe führen, entziehen sich aber weiterhin einer am Bedarf orientierten Planung. Dabei überlässt es der Gesetzgeber der Selbstverwaltung, den Schlüssel zu finden, mit dem eine Institutseinrichtung und eine Vollzeitarztstelle in das richtige Verhältnis für die Ermittlung einer gesättigten vertragsärztlichen Versorgung gesetzt werden. Am Beispiel der Psychiatrischen Institutsambulanzen zeigen sich nicht ausgeschöpfte Potenziale einer sektorübergreifenden Versorgungsplanung. Obgleich Zulassungen für Ärzte und Psychotherapeuten mit vergleichbarem Leistungsangebot in gesperrten Planungsbereichen nicht mehr vergeben werden können, sind Ermächtigungen für die genannten Institute weiter ohne Einschränkung möglich.

Zukünftig unterstützen weitere Leistungserbringer durch Ermächtigung die ambulante Versorgung, sofern eine Unterversorgung besteht oder einzutreten droht. Das gilt auch bei lokalen Versorgungslücken, die in durchaus gut versorgten Gebieten auftreten. Ärzte in Reha- und stationären Pflegeeinrichtungen zählen dazu. Den KVen wird erlaubt, Eigeneinrichtungen in ambulant nicht ausreichend versorgten Gebieten zu betreiben. Deren Leistungen erhöhen den Versorgungsgrad ebenso, wie die Leistungen der ambulanten Spezialfachärztlichen Versorgung nach $\mathbb{S} 116 \mathrm{~b}$ SGB V, die von der oben beschriebenen spezialisierten fachärztlichen Versorgung zu unterscheiden ist. Für die spezialfachärztliche Versorgung verzichtet der Gesetzgeber auf eine Bedarfsplanung, erweitert aber den Landesausschuss um Vertreter der Krankenhäuser zu einem weiteren neuen Gremium ohne relevante Entscheidungsbefugnis. Dies prüft die Voraussetzungen von Leistungserbringern, die ihren Antrag zur Erbringung von Leistungen nach $\mathbb{S} 116 \mathrm{~b}$ SGB V für den stationären oder ambulanten Bereich einreichen.

Die Wirkungen der neuen Bedarfsplanung sind noch nicht konkret abschätzbar. Das kurzfristig größte finanzielle Risiko für die Krankenkassen liegt in den Punktwertzuschlägen außerhalb der Gesamtvergütung und dem Entfallen von Fallzahlbegrenzungs- und minderungsregelungen. Wenn für Planungsräume Unterversorgung besteht oder droht oder aber die Beteiligten einen zusätzlichen lokalen Versorgungsbedarf finden, der Sonderzulassungen rechtfertigt, werden diese Punktwertzuschläge vereinbart, sofern die vom Bewertungsausschuss zu beschließenden Kriterien für »förderungswürdige Leistungen « oder für »besonders zu fördernde Leistungserbringer« erfüllt sind. Weil den Ländern die Möglichkeit eröffnet wurde, von der Bedarfsplanungs-Richtlinie abzuweichen, ist es auch erlaubt, mehr Versorgungsbedarf festzustellen, als dies die Kassenärztlichen Vereinigungen im Einvernehmen mit den Krankenkassen nach den Vorgaben der Richtlinie errechnen würden. Obgleich die Länder lange und intensiv dafür gestritten haben, den tatsächlichen Versorgungsbedarf unter Berücksichtigung von Demografie und Morbidität zu ermitteln und sich kleinräumig zu orientieren, ist ihr Anliegen methodisch nicht einlösbar.

Die Modellrechnung für eine wohnortnahe hausärztliche Versorgung zeigt, welche Wirkungen die Vergütungszuschläge erreichen können. Wählt man einen Raumbezug für Hausärzte mit der Gebietsgröße von Gemeindeverbänden, entstehen 4.628 neue Planungsbereiche. Bis zu einem Versorgungsgrad von $110 \%$ erhöht sich der Bedarf auf 8.785 neue Sitze für Hausärzte. Aus Daten der AOKs lässt sich je nach Berechnungsmethode die Wirkung für die Punktwertzuschläge abschätzen. Etwa ein Drittel aller Planungsbereiche geraten in diesem Modell bei einem Schwellenwert von $75 \%$ des Versorgungsbedarfs vom ersten Tag des Inkrafttretens der neuen Richtlinie in die Unterversorgung. Rund ein Achtel aller Hausärzte sind in diesen Planungsbereichen bereits niedergelassen. Wird die Schwelle für Unterversorgung auf $90 \%$ angehoben, ist die Hälfte aller Planungsbereiche mit etwa einem Drittel aller niedergelassenen Hausärzte betroffen. Die neuen Praxen werden mit Sicherstellungszuschlägen und Mitteln aus dem geplanten Strukturfonds ausgestattet, sofern sich Bewerber finden. Ärzte in diesen Regionen profitieren von Punktwertzuschlägen und werden von Maßnahmen zur Fallzahlbegrenzung oder -minderung ausgenommen - solange Unterversorgung besteht. Ähnliche Modelle können für Regionen mit lokalem Versorgungsbedarf gerechnet werden. Wie viele Ärzte also insgesamt profitieren, lässt sich noch nicht abschätzen.

Für die fachärztliche Versorgung werden je nach Bezugsjahr und Bezugsraum zwischen 5.000 und 16.500 Neuzulassungen diskutiert, obgleich bislang von einem erheblichen Mangel keine Rede war. Eine weitere Steigerung verspricht die neu zu gestaltende Zulassung wegen Sonderbedarf, die in bereits gesperrten Regionen Niederlassungen ermöglicht und schon heute enorme regionale Versorgungsunterschiede verursacht. Die Honoraranpassungen folgen diesem Szenario unweigerlich. Die Lösung kündigt sich als sukzessive Abspaltung (spezial-)fachärztlicher Vergütung aus der Gesamtvergütung an, die neue Bedarfsplanung bereitet dafür den Weg im kollektiven Vertrag und lässt die Krankenkassen außen vor.

Bedarf ist nicht allein als Funktion von medizinischem Fortschritt, demografischer Entwicklung oder veränderter In-

\section{So bleibt die Frage, wie eine am Bedarf orientierte Versorgung zukünftig aussehen kann, auch von dieser Regierung unbeantwortet.}

anspruchnahme zu ermitteln. So bleibt die Frage, wie eine am Bedarf orientierte Versorgung zukünftig aussehen kann, auch von dieser Regierung unbeantwortet. Das Gesetz nimmt mit einer Fülle von Einzelmaßnahmen viele Vorschläge aus der Diskussion zur Neugestaltung der Bedarfsplanung auf, wagt sich aber weder an eine sektorenübergreifende Planung und Gestaltung der Versorgung noch an geeignete Konzepte heran. Zugunsten der Freiberuflichkeit vergibt die Regierungskoalition die Chancen, die in neuen Betriebs- und Organisationsformen liegen. Vertragliche Optionen zur Bedarfssteuerung durch die Kassen spielen keine Rolle. Die ursprüngliche 
Zielsetzung der 1993 mit dem Gesundheitsstrukturgesetz ${ }^{7}$ entwickelten Bedarfsplanung richtete sich auf die Steuerung von Zulassungen zur Vermeidung einer Ärzteschwemme. Diese Systematik wird zwar im Versorgungsstrukturgesetz beibehalten und zum Teil sinnvoll ergänzt. Jedoch werden mit dem Neuzuschnitt der Planungsräume die Versorgungslücken nicht nur transparenter, sondern als Folge von neuen und kleineren Räumen auch zahlreicher. Ob die neuen Anreize zur Niederlassung von Einzelpraxen auf dem Lande geeignet sind, um die Lücken als Ergebnis einer verfehlten Planung und
Verteilung zu schließen, bleibt fraglich. Für die Versorgung der Bevölkerung in der Fläche sind Versorgungskonzepte gefragt, die weit über die Stärkung der Freiberuflichkeit einer Berufsgruppe und eindimensionaler Klientel- und Anreizpolitik hinausreichen. Vom »Landärztegesetz « profitieren erst einmal Ärzte, die bereits jetzt die Versorgung sicherstellen. Ihre Arbeit wird zusätzlich honoriert.

7 Gesetz zur Sicherung und Strukturverbesserung der gesetzlichen Krankenversicherung (Gesundheitsstrukturgesetz, GSG), 21.12.1992

\section{Soziale Selbsthilfe im Gesundheitswesen}

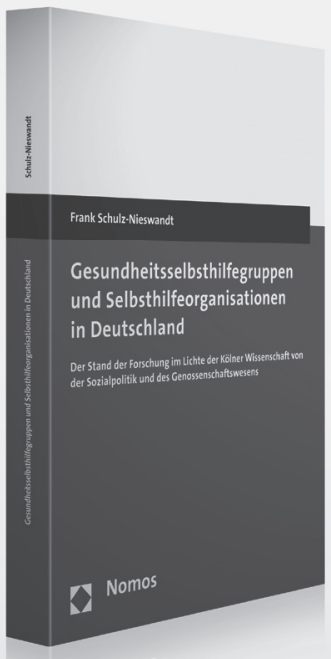

Als Teil des bürgerschaftlichen Engagements und des Dritten Sektors sind soziale Gesundheitsselbsthilfegruppen wenig erforscht. Das Buch gibt einen Überblick über den Forschungsstand und definiert den weiteren Forschungs- bedarf. Soziale Selbsthilfe wird genossenschaftsartig als selbstorganisierte Hilfe auf der Basis der Gegenseitigkeit verstanden.

\section{Gesundheitsselbsthilfegruppen und Selbsthilfeorganisationen in Deutschland}

Der Stand der Forschung im Lichte der KöIner Wissenschaft von der Sozialpolitik und des Genossenschaftswesens

Von Prof. Dr. Frank Schulz-Nieswandt 